\title{
Toward a Global Perspective of Sales Strategy Components
}

\author{
Xavier C. Martin
}

\begin{abstract}
There is a global consensus today on the relevance to consider the strategic role of sales departments, at least in BtoB. Nevertheless, to our knowledge and recently, only two empirical research studies have proposed what could be the components of a sales strategy (SS). The choices of these components are based only on salespeople's interviews in one case, and on convenience choices in the second one. The authors conclude that their measures of sales strategy need to be further validated and established.

The contribution of the present research is to broaden the perspective of SS to consider more congruent components, by considering some conceptual contributions from the strategic literature.

SS can be associated with the concepts of operational strategy, the Balanced Score Card, the questioning approach of strategy, and the customer approach of strategy. These approaches confirm the pertinence of considering these three dimensions of SS (clustering, organization structure and relational objectives/ selling models), even though they suggest some sub-dimensions of the three main components considered by the sales strategy literature. Moreover, a fourth dimension is proposed to foster a holistic view of a SS.

Empirically, 22 in-depth interviews were conducted with Sales Managers in France. Even though great discrepancy was noticed in the responses, these dimensions were mentioned frequently. Our next step will be to quantitatively validate these components.
\end{abstract}

\footnotetext{
X.C. Martin $(\bowtie)$

Novancia Business School, Paris, France

e-mail: xmartin@novancia.fr
}

L. Petruzzellis, R.S. Winer (eds.), Rediscovering the Essentiality of Marketing, Developments in Marketing Science: Proceedings of the Academy of Marketing Science, DOI 10.1007/978-3-319-29877-1_151 